\title{
Putting an Experience into Words: A Qualitative Investigation of Egyptian Drug Users' Perceptions of Expressive Writing
}

\author{
Amira Mohammed Ali* \\ Department of Psychiatric Nursing and Mental Health, Faculty of Nursing, Alexandria Universit, Egypt
}

Submission: June 27, 2018; Published: July 05, 2018

*Corresponding author: Amira Mohammed Ali, Department of Psychiatric Nursing and Mental Health Faculty of Nursing, Alexandria University, EdmonFremon St Smouha, Alexandria 36741, Egypt, Tel: +2-03-4291-578; Email: mercy.ofheaven2000@gmail.com

\begin{abstract}
Psychiatric treatments should be culturally adapted in order to bring desired outcomes. Though expressive writing (EW) has been a widely used intervention since it was first introduced more than 30 years ago; perceptions associated with use of EW among Arab participants have not been explored till the moment. In this study, a focus group discussion was used to illicit perceptions of inpatient drug users about EW and barriers to successful use of this modality. Negative attitudes toward disclosure and alexithymia were noted as key barriers to use of EW. Participants suggested that modifications of the intervention either being in a therapist-facilitated format or a group format might maximize the use and the benefits of EW.
\end{abstract}

Keywords: Alexithymia; expressive writing; substance-related disorders; cultural barriers

\section{Introduction}

Expressive writing (EW) is a narrative intervention that allows individuals to get a meaning of their painful experiences by writing down the deepest thoughts and emotions related to these experiences. It has been extensively used to produce physical and psychological health benefits [1]. Mechanisms that explain the therapeutic effects of EW are still unclear, but it is thought that EW works in a fashion similar to exposure therapies that act through desensitization frequent and detailed disclosure of unpleasant experiences through writing may result in habituation and extinction of trauma related thoughts and emotions. It is also postulated that cognitive processing and restructuring of the trauma-related schema takes place when people produce coherent narratives of their stressful events [2] Individuals' ability to disclose full details of a painful experience is affected by several individual characteristics such as level of emotional intelligence and gender [2,3]. In addition, the literature documents that people's degree of expressiveness varies from culture to another [4,5]. Nevertheless, most EW reports come from western countries; little is known about the use of EW in Arab samples.

\section{Methods}

Participants of the current study were hospitalized drug users that were formerly recruited in a larger EW controlled trial [6]. Interventions conducted during the time of the trial consisted of two didactic sessions about emotional disclosure (benefits of emotional disclosure, how denial and defensiveness contribute to continuation of drug abuse, and training on how to get in touch with one's thoughts and emotions) in addition to five 15-minutes sessions of EW that were delivered on a daily basis. The researcher read Pennbakers's instructions loudly, gave participants a copy of the instructions, advised them to write about the same topic every time or about different topics, and instructed them to keep diaries anonymous. Diaries were collected by the researcher and kept in a sealed envelope; participants were assured that no one can access their diaries either within or outside the unit. Many participants dropped out of the EW group, and most attrition took place during the EW phase. More, some participants refused to hand their diaries while some others handed the diaries blank. A check of random samples of the diaries revealed that most topics were addressed superficially (e.g., I was in love with someone and my family prevented our marriage); the emotional and thought contents in the written stories were revealed in a few cases only. The aim of this study was to identify barriers to successful EW among inpatient drug users.

Data Collection: After termination of the program, a focus group discussion was conducted to investigate participants' perceptions of EW as a therapy and to explore their personal experience of and barriers to using EW to describe traumatic 
events related to their drug use. The discussion was guided by a semi-structured protocol which involved three main topics: a) participants' experience of EW use b) difficulties encountered during writing and barriers to EW, and c) participants' suggestions to enhance the EW procedure.

Participants: This qualitative study used a convenience sample that comprised seven participants (who were still available in the unit after the conclusion of the writing trial, and who accepted to take part in the focus group). Participants were all males, and their age ranged between 19 and 42 years.
Qualitative Analysis: Since recording was not allowed by the unit rules, interviews were instantly transcribed by the researcher. An inductive thematic analysis approach $[7,8]$ was used to identify themes in the dataset where themes were summarized into bullet points with supporting quotations. For presentation of the data, quotations of the obtained themes were translated from Arabic to English.

\section{Results}

Table 1 illustrates common themes extracted from the group discussion accompanied by relevant quotes.

Table 1: Themes of the discussions indicating participants' perceptions of expressive writing, barriers, and recommendations for intervention improvement.

\begin{tabular}{|c|c|}
\hline Theme & Illustrative quotes \\
\hline Experience of expressive writing & $\begin{array}{l}\text { 'It is the first time I try to look deep into myself' } \\
\text { 'I was unfamiliar with this technique' } \\
\text { 'I know that many people fail the fourth step of the NA program because of writing, and now I } \\
\text { understand why writing can be such a problem' }\end{array}$ \\
\hline \multicolumn{2}{|l|}{ Barriers to expressive writing } \\
\hline Concerns about unsafe disclosure & 'I was insecure, I expected that you will read my story, and then I will regret that, so I could not let go' \\
\hline Doubts about the helpfulness of EW & $\begin{array}{l}\text { 'I took it lightly, not thinking that writing is going to help' } \\
\text { 'My problems are bigger than to be resolved by writing' }\end{array}$ \\
\hline Difficulty-initiating writing & $\begin{array}{l}\text { 'When thinking of an event to write about, many things came to my mind, my head was so crowded, and } \\
\text { it was hard to choose a topic for writing' } \\
\text { 'How to initiate the writing was the most difficult part' } \\
\text { 'My head was empty. I tried to squeeze it to write something, and the } 15 \text { minutes of the writing were too } \\
\text { long than I ever thought' }\end{array}$ \\
\hline Difficulty-naming feelings and thoughts & $\begin{array}{l}\text { 'It was easy to narrate a traumatic experience; however, I could not capture associated emotions and } \\
\text { thoughts' }\end{array}$ \\
\hline Writing-related emotional response & $\begin{array}{l}\text { 'I had many things to tell. I wrote too much. I read the diary over and over that I was so anxious. Instead } \\
\text { of feeling relieved, I felt tenser than before. I became hesitant to write again' }\end{array}$ \\
\hline Maximizing capacity of disclosure & $\begin{array}{l}\text { 'Individual discussion with a therapist may help me to disclose a really traumatic experience } \\
\text { appropriately' } \\
\text { 'Instead of directing us to choose any traumatic experience the writing instructions should be more } \\
\text { directive and structured in a way that facilitate exploration of a certain type of events' } \\
\text { 'Group discussion of the written content pertaining to traumatic events with colleagues might help me } \\
\text { express held emotions and explore hidden parts of the experience! }\end{array}$ \\
\hline
\end{tabular}

\section{Perceptions of the Experience of using EW}

All participants, except one, indicated that EW was a new and a difficult experience. EW entails participants to recall a trauma and describe their deepest thoughts and emotions about it. An attempt to consciously recall one's dumped traumas and to sensibly watch oneself and have a closer look at how he felt and thought about these traumas seemed to be a hard task for the subjects of this study.

\section{Barriers to Use of EW}

Three main themes related to barriers to use of EW were identified from the dataset: contextual evaluations, perceptions of personal ability to perform the writing task, and writing-related emotional response.

\section{Contextual Evaluations}

This theme involves participants' negative attitudes toward the EW therapy and the context in which it took place. Two subthemes evolved under this theme: concerns about unsafe disclosure and doubts about the therapeutic helpfulness of EW.

Concerns about Unsafe Disclosure: Despite the fact that participants were instructed to keep their diaries anonymous and that they were assured that no one would access their diaries, some participants thought that the researcher may check the 
diaries and know about their personal details. Participant noted that they may regret truthful letting go and that they feel awkward when others know about their "dirty actions".

Doubts about the therapeutic helpfulness of EW: Participants were chronic multiple drug users; they implied that they tried several types of treatments before-including invasive ones such as a subcutaneous capsule. Given their past experience of failure of such sophisticated treatments, participants felt that EW (in such a simple form of merely writing details of a painful experience) may not have any valuable benefits to offer as a therapy.

\section{Perceptions of Personal Ability to Perform the Writing Task}

This theme is concerned with individual barriers to use of EW. It describes participants' perceptions of their lack of skills needed to initiate and to pursue the writing process according to Pennbaker's instructions that were read at the start of the writing session. Two subthemes emerged under this theme: difficultyinitiating writing and difficulty-naming feelings and thoughts.

Difficulty-Initiating Writing: Some of the participants reported that it was hard to start writing for several reasons: some indicated that they had a crowd of racing thoughts about trauma topics that coming to choose a traumatic topic for their writing was hard. Others indicated a hardship to put the pen on the paper and start the writing while some others noted that they had no thoughts at all as if their heads were empty.

Difficulty-naming feelings and thoughts: This theme reflects a limitation in one of the aspects of emotional intelligence which involves the ability to identify one's emotions. Some participants indicated that describing their thoughts and feelings was the most difficult part of the writing process. It was possible for the participants to enumerate the details of their traumatic events but they lacked the ability to name the feelings which they had-it was also hard to differentiate between feelings and thoughts.

\section{Writing-Related Emotional Response}

This theme refers to the occurrence of undesirable emotional consequences after the writing procedure. One participant indicated that he went through the diaries over and over which was associated with a high feeling of distress that discouraged further writing. Though it was only one participant that reported encountering negative emotions, it is necessary to document this report since the study comprised only seven participants and those who wrote their deep emotions may have been very few.

\section{Discussion}

Drawing on the feedback derived from the study participants, EW was a hard activity for Egyptian drug users. Barriers to writing involved negative attitudes toward EW as an effective therapy and negative perceptions of participants' ability to perform the writing activity. Culture can play a role in the perception and response of individuals to EW. Inability to express emotions in this sample might have originated from the common social norms. Expression of emotions denoting vulnerability is highly discouraged in Egypt [9]. Therefore, it may be hard for people who believe that they should be in control of their emotions to realize that writing thoughts and emotions that reflect their "weaknesses" can be useful. We can further claim that participants' negative attitudes were signaled in their worries about the safety of disclosure in EW, despite the anonymity of the diaries. Research suggests that drug users have difficulties processing emotions and that alexithymia (a trait that involves inability of a person to discuss inner experiences and identify or disclose emotions) may contribute to substance dependence [10]. Alexithymia is a culture-bound construct [5]. There is some evidence that Egyptians have limited levels of emotional awareness and expressivity [11]. Moreover, alexithymia is prevalent among Egyptian drug users, and it is associated with polysubstance use, fewer hospitalizations, and treatment discontinuation [12]. Although not objectively assessed, alexithymia was vivid in participants' reports-both inability to disclose an experience or name their feelings (Table 1). Lack of insight of feelings and thoughts might explain why most narratives focused on external factors (e.g., the family that prevented the marriage) rather than on person's cognitive and emotional reactions to the event. In accordance with this finding, an earlier quantitative EW study reported that Asian Americans used less insight words and demonstrated less improvement of distress symptoms after writing compared with European Americans [4] Research documents that negative emotions increase immediately after EW, but negative emotions vanish before the next day [1] One participant, however, denoted that he read the diary repeatedly, which was followed by an intense state of distress that he decided not to write again. The researcher could not find similar reports in the available literature, but it seems that reading diaries (which probably involved unprocessed trauma related thoughts and emotions) repetitively might have flared up ruminations about the emotionally charged experience, which subsequently increased the level of distress. It seems that use of EW solo as a self-help therapy may not be fruitful in this group. Participants suggested that they might be more able to identify and discuss their thoughts and emotions if the procedure was done verbally with the help of a therapist or in a group format.

\section{Limitations and Strengths of the Study}

This study has some limitations. Findings may not be generalized since the number of participants was limited, and participants were only males-it is well-known that emotional expressivity is associated with gender differences. Generalizability, however, was not the main aim of this study which has the merit of being the first attempt to explore the perceptions and experience of using EW by Arab participants.

\section{Conclusion}

We can conclude that negative attitudes toward emotional expression and alexithymia were key barriers to effective use of EW. Future studies involving disclosure interventions in Egyptian 
drug users should be culturally adapted, facilitated by a therapist, and embracing components that address alexithymia and negative attitudes toward emotional disclosure.

\section{References}

1. Meshberg Cohen SD, Svikis, TJ McMahon (2014) Expressive Writing as a Therapeutic Process for Drug Dependent Women. Subst Abus 35(1) 80-88.

2. Ali AM (2018) The effect of expressive writing on illicit drug users: A meta-analytic review. Under review.

3. Ali AM (2018) The relationship between emotional intelligence, distress disclosure, and psychological distress among Egyptian illicit substance users. Journal of Addiction and Recovery 1(1002): 1-5.

4. Knowles ED, JR Wearing, B Campos (2011) Culture and the Health Benefits of Expressive Writing. Soc Psychol Person Sci 2(4): 408-415.

5. Fukunishi I (1997) Is Alexithymia a Culture-Bound Construct? Validity and Reliability of the Japanese Versions of the 20-Item Toronto Alexithymia Scale and Modified Beth Israel Hospital Psychosomatic Questionnaire. Psychological Reports 80(3): 787-799.
6. Ali AM (2017) The effect of expressive disclosure writing on selfstigma, depression, and anxiety among drug users in a governmental hospital in Egypt: A non-randomized controlled trial. J Depress Anxiety Disord 1(1): 31-40

7. Bernard H, Ryan G (1998) Handbook of methods in cultural anthropology. Text analysis: Qualitative and quantitative methods.

8. Maguire M, B Delahunt (2017) Doing a Thematic Analysis: A Practical, Step-by-Step Guide for Learning and Teaching Scholars. All Ireland Journal of Teaching and Learning in Higher Education (AISHE-J) 8(3): 3351-33514.

9. Krieger L (1989) Nerves and psychosomatic illness: the case of Um Ramadan. Health Care Women Int 10(2-3): 181-93.

10. Morie KP (2016) Alexithymia and Addiction: A Review and Preliminary Data Suggesting Neurobiological Links to Reward/Loss Processing Current Addiction Reports 3(2): 239-248.

11. Trovato G (2013) Cross-Cultural Perspectives on Emotion Expressive Humanoid Robotic Head: Recognition of Facial Expressions and Symbols. International Journal of Social Robotics 5(4): 515-527.

12. El Rasheed AH (2001) Alexithymia in Egyptian Substance Abusers Subst Abus 22(1): 11-21.

\section{Your next submission with Juniper Publishers} will reach you the below assets

- Quality Editorial service

- Swift Peer Review

- Reprints availability

- E-prints Service

- Manuscript Podcast for convenient understanding

- Global attainment for your research

- Manuscript accessibility in different formats

( Pdf, E-pub, Full Text, Audio)

- Unceasing customer service

Track the below URL for one-step submission https://juniperpublishers.com/online-submission.php 